\title{
Health Canada to increase transparency
}

Cite as: CMAJ 2017 April 10;189:E548. doi: 10.1503/cmaj.1095409

H ealth Canada plans to increase transparency into its regulatory process for drugs and medical devices by publicly releasing clinical information submitted by companies who receive market authorization.

"Opening access to clinical information used to support the authorization has widespread health system benefits and can help Canadians make informed decisions about their health," states a Health Canada report.

Drug companies and medical-device makers submit this information, which includes clinical trial data, to support the safety and effectiveness of their products. It is now considered confidential business information.

Under the proposed transparency policy, the information will be kept confidential during the regulatory process but disclosed if the drug or medical device is approved for sale. According to Health
Canada, this information is "far more comprehensive" than data available in sources such as medical journals and clinical trial registries.

Companies will be able to request, however, that certain parts of the clinical information they submit be exempt from public release. Health Canada is working with industry to determine what types of information will be eligible for exemption. Any information that could potentially identify clinical trial participants would also be removed.

Health Canada states that this transparency initiative will increase public confidence in the drug and medical device review process; help patients and health care providers make more informed decisions about drugs and medical devices; avoid duplication in clinical trials; and make clinical trial data available for secondary analysis to advance medical science.
The report notes that the European Medicines Agency (EMA) and the United States Food and Drug Administration (FDA) have also recently introduced similar initiatives to share clinical information while still protecting the commercial interests of companies.

"Health Canada intends to develop draft regulations based on the approach described in this paper, and will consult stakeholders on implementation of the proposed approach, including processes for review and redaction, mechanisms for public release of clinical information, and safeguards against commercial use," the report concludes. "Once in force, the regulations will establish a new channel for public access to clinical information."

Health Canada will accept public feedback on the proposed policy until May 26.

Roger Collier, CMAJ 